\title{
Solvent-Free Synthesis of Carboxylic Acids and Amide Analogs of CAPE (Caffeic Acid Phenethyl Ester) under Infrared Irradiation Conditions
}

\author{
Pablo A. Martínez-Soriano ${ }^{*}$, José R. Macías-Pérez², Ana María Velázquez¹, \\ Brígida del Carmen Camacho-Enriquez ${ }^{1}$, Gustavo Pretelín-Castillo',

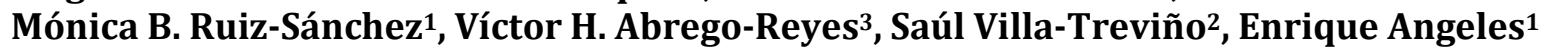 \\ ${ }^{1}$ Laboratorio de Química Medicinal y Teórica, Departamento de Ciencias Químicas, FES Cuautitlán, \\ Universidad Nacional Autónoma de México, Cuautitlán Izcalli, México \\ ${ }^{2}$ Departamento de Biología celular, Centro de Investigación y Estudios Avanzados, Instituto Politécnico \\ Nacional, México, D.F., México \\ ${ }^{3}$ Qsar Analytics S.A. de C.V. Ciudad Satélite, Naucalpan de Juárez, México \\ Email: ${ }^{*}$ arturin sirio@yahoo.com.mx, angeles@unam.mx
}

Received 19 March 2015; accepted 30 April 2015; published 4 May 2015

Copyright @ 2015 by authors and Scientific Research Publishing Inc.

This work is licensed under the Creative Commons Attribution International License (CC BY).

http://creativecommons.org/licenses/by/4.0/

(c) (i) Open Access

\begin{abstract}
A convenient and easy method is described for the formation of carboxamides from carboxylic acids and primary amines in solventless conditions using infrared (IR) light. Thus, under IR light, cinnamic acid derivatives and amines can produce yields ranging from $50 \%$ to $85 \%$ of the resulting amide.
\end{abstract}

\section{Keywords}

Amides, Carboxylic Acids, Amines, Infrared Light, Solventless, CAPE, CAPA, Cinnamic Acid Analogs

\section{Introduction}

Caffeic acid phenethyl ester (CAPE) is a natural phenolic chemical compound found in a variety of plants. It is also a component of propolis from honeybee hives, used as a sealant and to keep the hive clean from fungus and other contaminants [1].

Several in vitro pharmacokinetic effects have been reported for CAPE, including a positive effect on reducing

${ }^{*}$ Corresponding author.

How to cite this paper: Martínez-Soriano, P.A., et al. (2015) Solvent-Free Synthesis of Carboxylic Acids and Amide Analogs of CAPE (Caffeic Acid Phenethyl Ester) under Infrared Irradiation Conditions. Green and Sustainable Chemistry, 5, 81-91. http://dx.doi.org/10.4236/gsc.2015.52011 
carcinogenic incidence by boosting cytoprotection against oxidative stress. Antimitogenic, anticarcinogenic, anti-inflammatory and immunomodulatory properties have also been reported [2]. CAPE has been shown to suppress acute immune and inflammatory responses and to hold promise for therapeutic anti-inflammatory applications activating certain enzymatic systems that have antioxidant properties [3]. An anti-cancer effect was observed when mice skin, exposed to TPA to induce skin papillomas, was then treated with bee propolis; CAPE significantly reduced the number of papillomas [4] [5].

The purpose of this study was to synthesize a series of CAPE amide derivatives and previously reported CAPE analogs via a solvent-free procedure. Previous studies of caffeic acid phenethyl amides (CAPA) note that it can act as an antioxidant against lipid peroxidation [6] as well as a potential anti-inflammatory agent through inhibition of 5-lipoxygenase [7]. Using a 2,2-diphenyl-1-picrylhydrazyl assay, CAPA has also been shown to exhibit significant radical scavenging activity [8]. Although various CAPA analogs have been investigated both for radical scavenging activity and for $\alpha$-glucosidaseinhibition [9], new entities with other functionalities have not yet been studied. Tests have shown CAPA to have stronger in vitro cytoprotective effects than CAPE [10].

Amides are of considerable interest in a number of areas, from drug discovery to polymer industry, and therefore their synthesis has been, and remains, a topic of significant interest for organic chemists. The synthesis of these compounds involves a transformation of general synthetic interest that often requires harsh conditions (high temperatures and long reaction times) or the use of often highly toxic catalysts or reagents.

While there are few reports of amide synthesis from cinnamic derivatives, there are some reports that amides derived from cinnamic acids have antioxidant, anti-atherogenic, antiviral, cytotoxic and antifungal properties, with sundry action mechanisms from enzyme inhibition to free radical scavenging. The synthesis of these compounds from different functional groups has also recently been described by different authors, who have synthesized them from ketones (hidrazoic acid [11] [12], via Beckmann rearrangement [13], and via azido-Schmidt reaction with $\mathrm{FeCl}_{3}$ [14]); from aldehydes (aldehydes with alkyl azides [15], oxidative amination with amines [16], oxidative amination catalyzed by zinc [17], and oxidative amination catalyzed by palladium with hydrogen peroxide [18]); from acid halides via Schotten-Baumann reaction [19]; from carboxylic acid (with amines and carbodiimides [20]); using amines and a molecular sieve [21]; from amines with nanosulfated titanium dioxide [22]; using epimerization-prone carboxylic acids and amines with T3P and Pyridine [23]; using triacyloxyboranes [24]; using trimethylaluminium [25]; with tosyl chloride in solventless conditions [26]; with urea using microwaves [27]; using solid phase synthesis with polymer bound reagents [28]; using isonitriles [29]; from esters and lactones [30]; from imines [31]; and from esters (assisted by potassium tert-butoxide [32], with magnesium nitride [33], and from methyl ketones or carbinols [34]).

On this occasion and as a part of the research agenda of the bioactive molecules program, we report the synthesis of amides from cinnamic acid analogs and from phenylacetic acid with aliphatic amines in the absence of solvent. We also report the synthesis of cinnamic acid derivatives. Some of these amides-compounds 10 and 11 (see Table 2) —have presented biological activity against liver cancer [10]. The use of infrared light as an alternate source of energy allows reactions to be faster as it shortens the reaction time, thus the heating is faster. Although this does not compare to the use of microwaves, that allows much shorter reaction times, the cost of one IR lightbulb is much lesser, so minimizing costs is a priority now. We use as energy source a $300 \mathrm{~W}$ infrared lightbulb connected to a rheostat to control the amount of energy supplied to the reaction.

\section{Results and Discussion}

The CAPE analogs were synthesized using the methodology described in "experimental" section. Our method using IR energy allows us to prepare both amides and cinnamic acids analogs. Cinnamic acid analogs were prepared in good yields with short reaction times (see Table 1); this allows to an efficient preparation and purification. Our results are consistent with those reported on literature achieving in some cases better yields.

In Table 2, we show the molecules synthesized using our methodology, some of them have high yields, and others have very low yields. This increase of yield is due to the presence of halogens and phenoxy moieties in the acids and in the amines used to prepare these molecules. On the other hand nitro and methoxy moieties decreased yield. The presence of trifluoromethyl moiety diminished the yield in the presence of phenoxyacetic acid while with cinnamic acid result in good yields. 
Table 1. Prepared cinnamic acid analogs and yields.

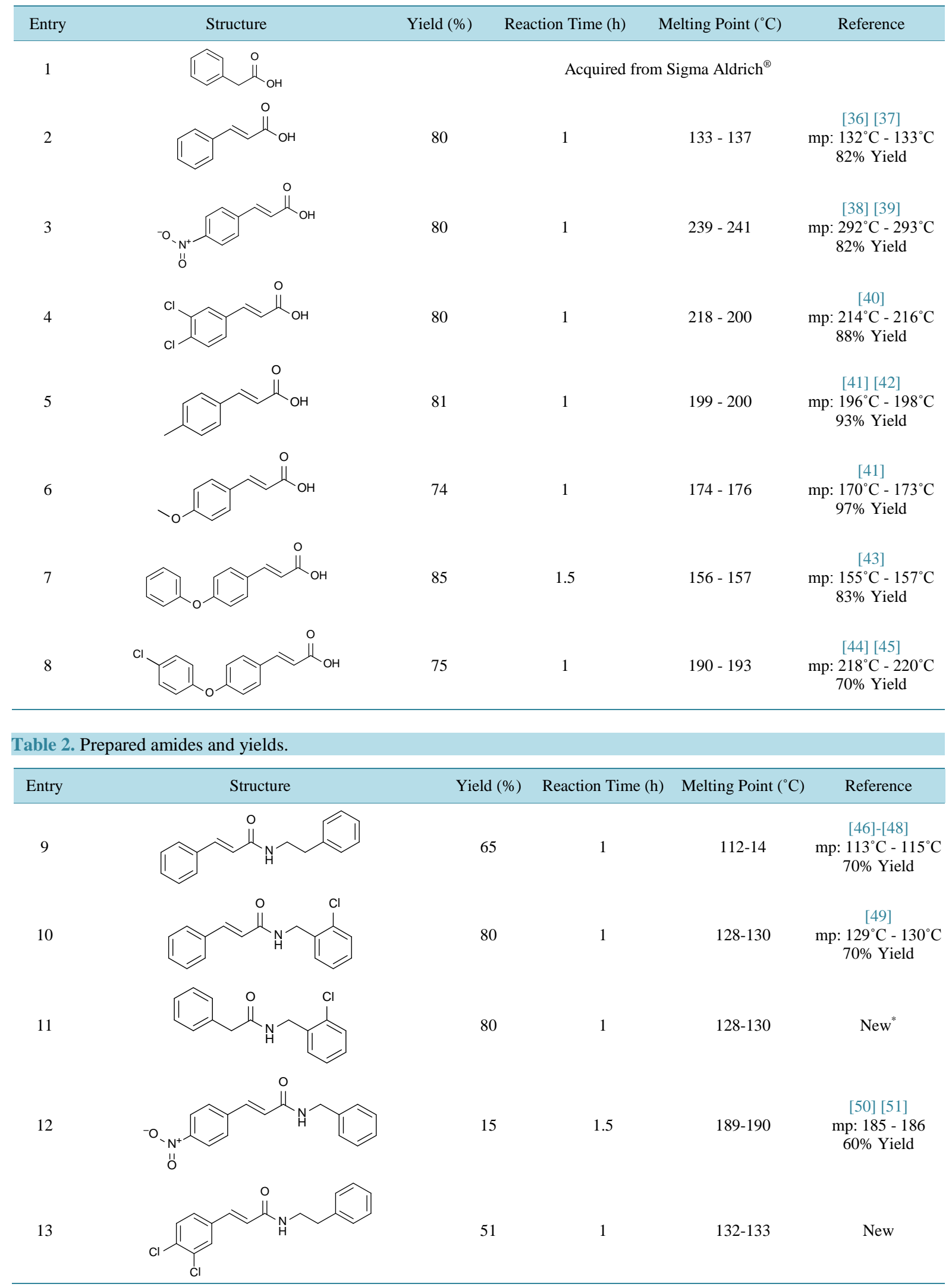




\section{Continued}

14<smiles>O=C(Cc1ccccc1)NCc1ccc(Cl)c(Cl)c1</smiles>

15<smiles>O=C(Cc1ccccc1)NCCc1ccccc1</smiles>

16<smiles>O=C(Cc1ccccc1)NCc1ccccc1</smiles>

17<smiles>O=C(/C=C/c1ccc(Oc2ccc(Cl)cc2)cc1)NCCc1ccccc1</smiles>

18<smiles>COc1ccc(/C=C/C(=O)NCCc2ccccc2)cc1</smiles>

19<smiles>O=C(/C=C/c1ccccc1)NCc1cccc(C(F)(F)F)c1</smiles><smiles>COc1ccccc1CNC(=O)/C=C/c1ccccc1</smiles>

21<smiles>COc1ccccc1CNC(=O)/C=C/c1ccc(Oc2ccccc2)cc1</smiles>

22<smiles>O=C(/C=C/c1ccc(Oc2ccccc2)cc1)NCc1cccc(C(F)(F)F)c1</smiles><smiles>O=C(Cc1ccccc1)NCc1ccc(F)cc1</smiles><smiles>O=C(Cc1ccccc1)NCc1cc(C(F)(F)F)cc(C(F)(F)F)c1</smiles>

23

24$$
\text { F } \overbrace{F}
$$

28
121-122

[52] [54] [55] mp: 118 92\%Yield mp: $128-131$ $60 \%$ Yield
102-104

$0.5 \quad 134-136 \quad 77 \%$ Yield
91-95

New 


\section{Continued}

25

26<smiles>O=C(Cc1ccccc1)NCc1cccc(C(F)(F)F)c1</smiles>

27<smiles>COc1ccc(/C=C/C(=O)NCc2ccccc2OC)cc1</smiles>

28<smiles>O=C(/C=C/c1ccc([N+](=O)[O-])cc1)NCc1ccc(F)cc1</smiles>

29<smiles>O=C(/C=C/c1ccc(Oc2ccccc2)cc1)NCc1ccc(F)cc1</smiles>

30<smiles>O=C(/C=C/c1ccc(Cl)c(Cl)c1)NCc1ccc(F)cc1</smiles>

31<smiles>O=C(/C=C/c1ccc(Oc2ccccc2)cc1)NCc1cc(C(F)(F)F)cc(C(F)(F)F)c1</smiles>

32<smiles>Cc1ccc(/C=C/C(=O)NCc2ccc(F)cc2)cc1</smiles>

*This compound's first synthesis is reported in this paper, the reference [10] is for biological activity.

Our working group proposed the synthesis of amides from carboxylic acids and amines without the presence of solvent and using infrared energy. This method has facilitated the production of the amides listed above using a relatively clean procedure that generates few reaction by-products.

This procedure also eases the purification process, as what remains are only any unreacted raw materials and trace amounts of the by-products. This produces good yields and amides with a high degree of purity after separation treatment. This is also a low-cost procedure, because, as mentioned above, it does not require the use of expensive catalysts or solvents that must be recovered, nor any treatment is required as a result of their use.

\section{Conclusion}

This paper presents a novel methodology for the synthesis of cinnamic acid analogs via a Knövenagel-Döbner modification and CAPA amide analogs in the absence of solvent and with good yields, using infrared radiation as energy source.

\section{Experimental}

\subsection{Synthesis of Cinnamic Analogs}

Our team prepared the cinnamic acid analogs that were not commercially available-4-(4-chlorophenoxy) cin- 
namic acid and 4-phenoxy cinnamic acid—via a Knövenagel-Döbner condensation using piperidine and glacial acetic acid as catalyst and solvent, respectively [35] [51]. The other cinnamic acid analogs (cinnamic acid, 4-nitro cinnamic acid, 3,4-dichloro cinnamic acid, 4-methoxy cinnamic acid, and 4-methyl cinnamic acid) were also synthesized, onlyphenylacetic acid was purchased from Sigma Aldrich Co. ${ }^{\circledR}$ (Scheme 1).

The corresponding substituted benzaldehyde and malonic acid weighed on a 1:1 ratio were placed in a flask with piperidine (1 mL for each part of substituted benzaldehyde (ratio 1.25:1)) and glacial acetic acid (2.5 mL for each part of substituted benzaldehyde (ratio 1.77:1)). This flask was connected to the reflux apparatus at $140^{\circ} \mathrm{C}-160^{\circ} \mathrm{C}$, which is the temperature necessary to carry out the reaction, followed by TLC (hexane: EtOAc, 80:20). Upon completion of the reaction, ice or cold water was added to the flask until the acid precipitated; it was then filtered and washed repeatedly with water $(3 \times 100 \mathrm{~mL})$. Recrystallization from EtOAc produces the corresponding acid.

If the acid did not precipitate but formed an emulsion, liquid-liquid extraction with EtOAc $(5 \times 50 \mathrm{~mL})$ was performed. Following this step, some acetic acid was dissolved in the EtOAc. Brine $(3 \times 30 \mathrm{~mL})$ was then used to remove this acetic acid, and anhydrous sodium sulfate was then used to remove water from the organic phase (EtOAc) to allow recrystallization.

\subsubsection{General Procedure, for 3-(4-Phenoxyphenyl)-2-Propenoic Acid and} 3-[4-(4-Chlorophenoxy) Phenyl]-2-Propenoic Acid

4-(4-chlorophenoxy) benzaldehyde $(1 \mathrm{~g}, 3.640 \mathrm{mmol})$ and malonic acid $(1 \mathrm{~g}, 9.6097 \mathrm{mmol})$ were weighed in a round bottom flask; piperidine $(1 \mathrm{~mL}, 11.76 \mathrm{mmol})$ and glacial acetic acid $(2.5 \mathrm{~mL}, 41.66 \mathrm{mmol})$ were added. This was taken to the IR light at $130^{\circ} \mathrm{C}-140^{\circ} \mathrm{C}$. After 1 hour the reaction mixture was cooled and cold water with ice was added until precipitation. The flask was brought to room temperature and then filtered and rinsed with water at room temperature until no signs of the aldehyde were present. Sufficient AcOEt was added to dissolve the crystals, and anhydrous sodium sulfate was added to remove water captured by crystals. Recrystallization then occurred, with the appearance of white needles.

\section{1) 3-(4-phenoxyphenyl)-2-propenoic acid (7)}

Yield (85\%) mp $156^{\circ} \mathrm{C}-157^{\circ} \mathrm{C}$. IR (Diamond, $\left.\mathrm{cm}^{-1}\right)$ : $v_{\max } 1588,3031,3200-2200 .{ }^{1} \mathrm{H}$ NMR $\left(\mathrm{CDCl}_{3}, 300\right.$ $\mathrm{MHz}): \delta 6.30(1 \mathrm{H}, \mathrm{d}), 6.90(5 \mathrm{H}, \mathrm{m}), 7.24(4 \mathrm{H}, \mathrm{m}), 7.64(1 \mathrm{H}, \mathrm{d}, J=15.6 \mathrm{~Hz}), 8.95(1 \mathrm{H}, \mathrm{s}) ;{ }^{13} \mathrm{C} \mathrm{NMR}\left(\mathrm{CDCl}_{3}, 75\right.$ MHz): $\delta 116.02(-\mathrm{CH}=), 117.02,117.32,121.61,125.69,128.21,128.91(\mathrm{Ph}), 144.64(-\mathrm{CH}=), 155.32,156.13$ (Ph) $169.64(\mathrm{COOH})$.

\section{2) 3-[4-(4-chlorophenoxy) phenyl]-2-propenoic acid (8)}

Yield $(75 \%) \mathrm{mp} 190^{\circ} \mathrm{C}-193^{\circ} \mathrm{C}$. IR (Diamond, $\left.\mathrm{cm}^{-1}\right): v_{\max } 1570,3025,3200-2200 .{ }^{1} \mathrm{H}$ NMR $\left(\mathrm{CDCl}_{3}, 300\right.$ MHz): $\delta 6.41(1 \mathrm{H}, \mathrm{d}), 6.87(4 \mathrm{H}, \mathrm{m}), 7.25(4 \mathrm{H}, \mathrm{m}), 7.61(1 \mathrm{H}, \mathrm{d}, J=15.6 \mathrm{~Hz}), 8.45(1 \mathrm{H}, \mathrm{s}) .{ }^{13} \mathrm{C} \mathrm{NMR}\left(\mathrm{CDCl}_{3}, 75\right.$ MHz): $\delta 116.97(-\mathrm{CH}=), 117.22,118.73,125.69,126.94,128.21,128.91(\mathrm{Ph}), 145.70(-\mathrm{CH}=), 154.23,155.13$ (Ph) $171.78(\mathrm{COOH})$.

\subsection{Amide Synthesis}

The acid and amine, weighed on a 1:1 ratio, were placed in a flask and connected to the reflux apparatus at $140^{\circ} \mathrm{C}-160^{\circ} \mathrm{C}$ with the $300 \mathrm{~W}$ IR light bulb, followed by TLC(hexane: EtOAc, 50:50). Upon completion of the reaction, sufficient AcOEt was added to dissolve the reaction product.

A small amount of activated charcoal was added to the reaction mixture and filtered over diatomaceous earth (Hyflo Super Cel. ${ }^{\circledR}$ Diatomaceous earth) purchased from Sigma Aldrich ${ }^{\circledR}$ to remove the activated charcoal. Recrystallization from EtOAc produced the corresponding amide.

Further recrystallizations were made with an AcOEt: hexane 90:10 mixture only when necessary (Scheme 2).<smiles>[R]c1ccc(C=CC(=O)O)cc1</smiles>

R: phenoxy, 4-chlorophenoxy

a: piperidine, glacial acetic acid

Scheme 1. Knövenagel-Döbner condensation used to prepare cinnamic acid analogs. 


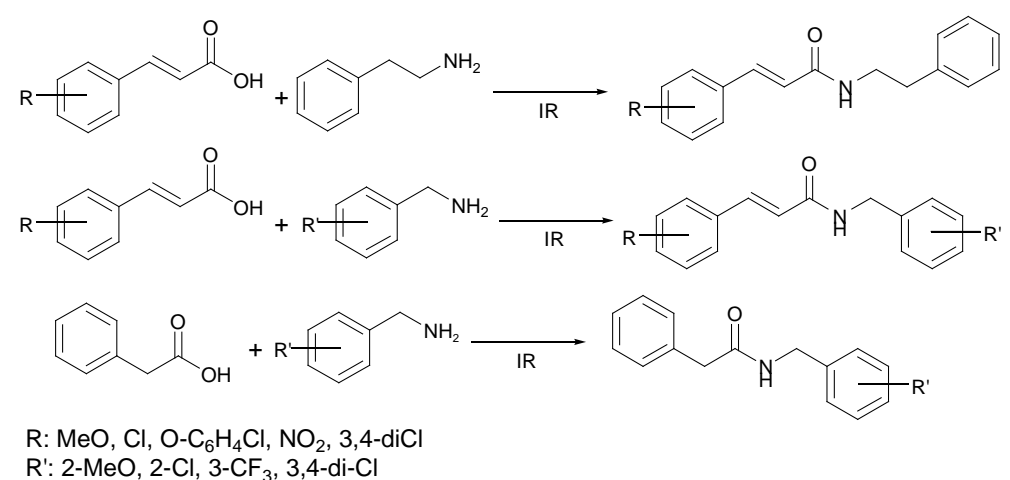

Scheme 2. Reactions involved on the preparation of amides.

\subsubsection{General Procedure for the Preparation of Amides (9-32). Exemplified by}

3-Phenyl-N-(2-Phenylethyl)-2-Propenamide

Cinnamic acid ( $0.5 \mathrm{~g}, 3.374 \mathrm{mmol})$ was weighed in a round bottom flask, and phenylethylamine $(0.5 \mathrm{~g}, 4.1261$ mmol) was added dropwise at $140^{\circ} \mathrm{C}-160^{\circ} \mathrm{C}$ without solvent. After 1 hour the reaction mixture was cooled to room temperature. AcOEt and active charcoal were added and filtered over diatomaceous earth (celite). Solvent was removed under reduced pressure until crystallization and recrystallized from a mixture of AcOEt and hexane.

*Only data for new amides are shown

1) N-(2-chlorobenzyl)-2-phenylacetamide (11)

Yield (3.9 g, 79.83\%); mp $118^{\circ} \mathrm{C}-120^{\circ} \mathrm{C}$. IR (Diamond, $\mathrm{cm}^{-1}$ ): $v_{\max } 1546,2923,3274 .{ }^{1} \mathrm{H}$ NMR $(300 \mathrm{MHz}$; $\left.\mathrm{CDCl}_{3} ; \mathrm{Me}_{4} \mathrm{Si}\right) \delta 3.63(2 \mathrm{H}, \mathrm{s}), 4.46(2 \mathrm{H}, \mathrm{d}, J=4.0), 5.90(1 \mathrm{H}, \mathrm{br}, \mathrm{s}, \mathrm{NH}), 7.29(9 \mathrm{H}, \mathrm{m}) ;{ }^{13} \mathrm{C} \mathrm{NMR}(75 \mathrm{MHz}$; $\left.\mathrm{CDCl}_{3} ; \mathrm{Me}_{4} \mathrm{Si}\right) \delta 42.05\left(\mathrm{CH}_{2}\right), 44.18\left(\mathrm{CH}_{2}\right), 127.44,127.85,129.28,129.48,129.88,129.89,130.32,133.91$, 135.05, 135.86 (Ph), 171.29 (-CO-).Analysis Calc. for $\mathrm{C}_{15} \mathrm{H}_{14}$ ClNO: C,69.3; H, 5.4; N, 5.3.Found C, 69.35; $\mathrm{H}$, $5.05 ; \mathrm{N}, 5.71$.

2) 3-(3,4-dichlorophenyl)-N-(2-phenylethyl)-2-propenamide(13)

Yield (2.55 g, 51\%), mp $132^{\circ} \mathrm{C}-133^{\circ} \mathrm{C}$ IR (Diamond, $\left.\mathrm{cm}^{-1}\right): v_{\max } 1550,2315,2920,3281 .{ }^{1} \mathrm{H}$ NMR (200 MHz; DMSO-d $\mathrm{d}_{6}$; Me $\left.\mathrm{M}_{4} \mathrm{Si}\right) \delta 2.79(2 \mathrm{H}, \mathrm{t}, J=7.4), 3.44(2 \mathrm{H}, \mathrm{m}), 6.72(1 \mathrm{H}, \mathrm{d}, J=15.8) .7 .43(8 \mathrm{H}, \mathrm{m}, \mathrm{Ph}), 7.85(1 \mathrm{H}, \mathrm{d}, J=$ 1.8), $8.29(1 \mathrm{H}, \mathrm{t}, \mathrm{NH}, J=11.2) ;{ }^{13} \mathrm{C}$ NMR (50 MHz; DMSO-d $\mathrm{d}_{6}$; Me $\left.{ }_{4} \mathrm{Si}\right) \delta 35.09\left(-\mathrm{CH}_{2}-\mathrm{Ph}\right), 124.49(-\mathrm{CH}=)$, 126.12, 127.27, 128.36, 128.66, 129.41, 131.01, 131.56, 131.68, 135.88, 136.00 (Ph), 139.41 (-CH=), 164.47 (-CO-). Calc. for $\mathrm{C}_{17} \mathrm{H}_{15} \mathrm{Cl}_{2} \mathrm{NO}$ : C, 63.7; H, 4.7; N, 4.3. Found C, 60.25; H, 4.71; N, 4.4.

\section{3) N-(3,4-dichlorobenzyl)-2-phenylacetamide (14)}

Yield $(1.51 \mathrm{~g}, 70 \%), \mathrm{mp} 132^{\circ} \mathrm{C}-133^{\circ} \mathrm{C}$. IR (Diamond, $\left.\mathrm{cm}^{-1}\right): v_{\max } 1534,2908,3265 .{ }^{1} \mathrm{H}$ NMR $(300 \mathrm{MHz}$; $\left.\mathrm{CDCl}_{3} ; \mathrm{Me}_{4} \mathrm{Si}\right) \delta 3.62(2 \mathrm{H}, \mathrm{s}), 4.32(2 \mathrm{H}, \mathrm{d}, J=6.1), 6.13(1 \mathrm{H}, \mathrm{s}, \mathrm{NH}), 7.23(8 \mathrm{H}, \mathrm{m}) ;{ }^{13} \mathrm{C}$ NMR $\left(75 \mathrm{MHz} ; \mathrm{CDCl}_{3}\right.$; $\left.\mathrm{Me}_{4} \mathrm{Si}\right) \delta 42.63\left(-\mathrm{CH}_{2}-\mathrm{CO}-\right), 43.98\left(\mathrm{CH}_{2}-\mathrm{Ph}\right), 127.59,128.36,129.98,130.12,130.22,131.39,132.17,133.44$, 135.51, 139.54 (Ph), 172.32 (-CO-). Calc. for $\mathrm{C}_{15} \mathrm{H}_{13} \mathrm{Cl}_{2} \mathrm{NO}$ : C, 61.2; H, 4.4; N, 4.7. Found C, 61.08; H, 3.81; N, 4.8 .

4) N-(2-phenylethyl)-3-[4-(4-chlorophenoxy)phenyl]-2-propenamide (17)

Yield $\left(0.37 \mathrm{~g}, 28 \%\right.$ ), $\mathrm{mp} 123^{\circ} \mathrm{C}-124^{\circ} \mathrm{C}$. IR (Diamond, $\left.\mathrm{cm}^{-1}\right): v_{\max } 1541,2922,3279 .{ }^{1} \mathrm{H}$ NMR (300 MHz; $\left.\mathrm{CDCl}_{3} ; \mathrm{Me}_{4} \mathrm{Si}\right) \delta 2.88(2 \mathrm{H}$, td, $J=6.9,6.8,2.5), 3.64(2 \mathrm{H}, \mathrm{qd}, J=6.9,2.5), 5.78(1 \mathrm{H}, \mathrm{t}, J=5.4), 6.26(1 \mathrm{H}, \mathrm{dd}, J=$ 15.6, 8.9), $7.16(13 \mathrm{H}, \mathrm{m}), 7.57(1 \mathrm{H}, \mathrm{dd}, J=15.6,8.6) ;{ }^{13} \mathrm{C}$ NMR $\left(75 \mathrm{MHz} ; \mathrm{CDCl}_{3} ; \mathrm{Me}_{4} \mathrm{Si}\right) \delta 35.67\left(\mathrm{CH}_{2}-\mathrm{Ph}\right)$, $40.87\left(\mathrm{CH}_{2}\right), 120.28$ (-CH=), 117.32, 126.62, 128.73, 128.81, 128.84, 129.49, 129.88, 129.93, 130.29, 138.83 (Ph), 140.17 (-CH=), 157.39, $155.90(\mathrm{Ph}), 165.58$ (-CO-). Calc. for $\mathrm{C}_{23} \mathrm{H}_{20} \mathrm{ClNO}_{2}$ : C, 73.1; H, 5.3; N, 3.7. Found C, 73.05; H, 4.62; N, 4.03.

\section{5) $\mathrm{N}$-[3-(trifluoromethyl)benzyl]-3-phenyl-2-propenamide (19)}

Yield (1.03 g, 50\%), mp $112^{\circ} \mathrm{C}-113^{\circ} \mathrm{C}$. IR (Diamond, $\mathrm{cm}^{-1}$ ): $v_{\max } 1588,2924,3261 .{ }^{1} \mathrm{H}$ NMR $(300 \mathrm{MHz}$; $\left.\mathrm{CDCl}_{3} ; \mathrm{Me}_{4} \mathrm{Si}\right) \delta 4.50(2 \mathrm{H}, \mathrm{d}, J=6.0), 6.40(2 \mathrm{H}, \mathrm{m}, \mathrm{NH}), 7.33(9 \mathrm{H}, \mathrm{m}), 7.58(1 \mathrm{H}, \mathrm{d}, J=15.6) ;{ }^{13} \mathrm{C}$ NMR $(75$ $\left.\mathrm{MHz} ; \mathrm{CDCl}_{3} ; \mathrm{Me}_{4} \mathrm{Si}\right) \delta 42.42\left(-\mathrm{CH}_{2}-\mathrm{Ph}\right), 124.13(-\mathrm{CH}=), 126.30,126.97,127.45,128.39,128.63,136.72,141.49$, 145.50, (Ph), 139.11 (-CH=), 164.26 (-CO-).Calc. for $\mathrm{C}_{17} \mathrm{H}_{14} \mathrm{~F}_{3} \mathrm{NO}$ : C, 66.8; H, 4.6; N, 4.5. Found C, 66.64; H, 4.03, N, 5.0. 
6) N-(2-methoxybenzyl)-3-phenyl -2-propenamide (20)

Yield (1.10 g, 61\%), mp $144^{\circ} \mathrm{C}-146^{\circ} \mathrm{C}$. IR (Diamond, $\mathrm{cm}^{-1}$ ): $v_{\max } 1538$, 2926, 3275. ${ }^{1} \mathrm{H}$ NMR $(300 \mathrm{MHz}$; $\mathrm{CDCl}_{3}$; $\left.\mathrm{Me}_{4} \mathrm{Si}\right) \delta 3.76(3 \mathrm{H}, \mathrm{s}), 4.48(2 \mathrm{H}, \mathrm{d}, J=5.9), 6.27(1 \mathrm{H}, \mathrm{brs}, \mathrm{NH}), 6.34(1 \mathrm{H}, \mathrm{d}, J=15.6), 6.82(2 \mathrm{H}, \mathrm{m})$, $7.27(7 \mathrm{H}, \mathrm{m}), 7.54(1 \mathrm{H}, \mathrm{d}, J=15.6) ;{ }^{13} \mathrm{C}$ NMR $\left(75 \mathrm{MHz} ; \mathrm{CDCl}_{3} ; \mathrm{Me}_{4} \mathrm{Si}\right) \delta 39.81\left(\mathrm{CH}_{2}-\mathrm{Ph}\right), 55.74\left(\mathrm{CH}_{3}\right), 121.54$ $(-\mathrm{CH}=), 111.03,121.74,127.02,128.62,129.62,129.76,130.40,130.73,135.83,158.56(\mathrm{Ph}), 141.79(-\mathrm{CH}=)$, 166.74 (-CO-).Calc. for $\mathrm{C}_{17} \mathrm{H}_{17} \mathrm{NO}_{2}$ : C, 76.3; H, 6.4; N 5.2. Found C, 76.38; H, 6.36; N, 5.26.

7) N-(2-methoxybenzyl)-3-(4-phenoxyphenyl)-2-propenamide (21)

Yield (1.06 g, 71\%), mp $123^{\circ} \mathrm{C}-124^{\circ} \mathrm{C}$. IR (Diamond, $\mathrm{cm}^{-1}$ ): $v_{\max } 1541$, 2946, 3289. ${ }^{1} \mathrm{H}$ NMR (300 MHz; $\mathrm{CDCl}_{3}$; $\left.\mathrm{Me}_{4} \mathrm{Si}\right) \delta 4.47$ (2H, d, $J=5.9$ ), $6.24(2 \mathrm{H}, \mathrm{m}), 6.87(6 \mathrm{H}, \mathrm{m}), 7.06$ (1H, d, $J=7.4$ ), 7.23 (6H, ddd, $J=19.2$, 16.3, 7.7), $7.50(1 \mathrm{H}, \mathrm{d}, J=15.6) ;{ }^{13} \mathrm{C}$ NMR (75 MHz; $\mathrm{CDCl}_{3}$; $\left.\mathrm{Me}_{4} \mathrm{Si}\right) \delta 39.55\left(\mathrm{CH}_{2}-\mathrm{Ph}\right), 55.39\left(\mathrm{CH}_{3}\right), 110.32$, $118.50(\mathrm{Ph}), 119.54(-\mathrm{CH}=), 120.77,123.96,126.27,128.93,129.40,129.83,129.89,129.94(\mathrm{Ph}), 140.16$ $(-\mathrm{CH}=)$, 156.37, 157.55, 158.78 (Ph), 165.81 (-CO-). Calc. for $\mathrm{C}_{23} \mathrm{H}_{21} \mathrm{NO}_{3}$ : C, 76.8; H, 5.8; N, 3.9\% Found C, 76.22; H, 5.61; N, 4.1.

8) $\mathrm{N}$-[3-(trifluoromethyl)benzyl]-3-(4-phenoxyphenyl)-2-propenamide (22)

Yield $(1.20 \mathrm{~g}, 73 \%), \mathrm{mp} 102^{\circ} \mathrm{C}-104^{\circ} \mathrm{C}$. IR (Diamond, $\mathrm{cm}^{-1}$ ): $v_{\max } 1562,3076,3266,1158 .{ }^{1} \mathrm{H}$ NMR (300 MHz; $\mathrm{CDCl}_{3}$; $\left.\mathrm{Me}_{4} \mathrm{Si}\right) \delta 4.62(2 \mathrm{H}, \mathrm{d}, J=6.0), 6.45(1 \mathrm{H}, \mathrm{d}, J=15.6), 6.61(1 \mathrm{H}, \mathrm{s}), 6.97(3 \mathrm{H}, \mathrm{d}, J=8.7), 7.08(2 \mathrm{H}$, d, $J=7.6), 7.2(1 \mathrm{H}, \mathrm{t}, J=7.4), 7.48(7 \mathrm{H}, \mathrm{m}), 7.68(1 \mathrm{H}, \mathrm{d}, J=15.6) ;{ }^{13} \mathrm{C} \mathrm{NMR}\left(75 \mathrm{MHz} ; \mathrm{CDCl}_{3} ; \mathrm{Me}_{4} \mathrm{Si}\right) \delta 43.24$ $\left(\mathrm{CH}_{2}-\mathrm{Ph}\right), 118.45(\mathrm{Ph}), 118.86(-\mathrm{CH}=), 119.64,\left(\mathrm{CF}_{3}, J=58.9\right), 124.09,124.32,124.33,124.36,124.38,129.21$, 129.41, 129.51, 129.97, 131.19, 139.43 (Ph), 141.07 (-CH=), 156.21, 159.11 (Ph), 166.31 (-CO-).Calc. for $\mathrm{C}_{23} \mathrm{H}_{18} \mathrm{~F}_{3} \mathrm{NO}_{2}$ : C, 69.5; H, 4.5; N, 3.5.Found C, 69.78; H,3.80; N, 3.9.

9) $\mathrm{N}$-[3,5-bis(trifluoromethyl)benzyl]-2-phenylacetamide (24)

Yield (0.37 g, 28\%), mp $91^{\circ} \mathrm{C}-95^{\circ} \mathrm{C}$. IR (Diamond, $\mathrm{cm}^{-1}$ ): $v_{\max } 1548,3068,3254,1117 .{ }^{1} \mathrm{H}$ NMR $(300 \mathrm{MHz}$; $\left.\mathrm{CDCl}_{3} ; \mathrm{Me}_{4} \mathrm{Si}\right) \delta 3.55(2 \mathrm{H}, \mathrm{s}), 4.49(2 \mathrm{H}, \mathrm{d}, J=6.0), 7.33(5 \mathrm{H}, \mathrm{d}, J=4.0), 7.91(2 \mathrm{H}, \mathrm{s}), 7.98(1 \mathrm{H}, \mathrm{s}), 8.80(1 \mathrm{H}, \mathrm{t}$, $\mathrm{NH}, J=5.8) ;{ }^{13} \mathrm{C}$ NMR (75 MHz; $\mathrm{CDCl}_{3}$; $\left.\mathrm{Me}{ }_{4} \mathrm{Si}\right) \delta 42.07\left(\mathrm{CH}_{2}-\mathrm{Ph}\right), 43.17\left(\mathrm{CH}_{2}-\mathrm{Ph}\right), 121.71\left(\mathrm{CF}_{3}\right), 122.78$, 126.42, 127.75, 129.05, 129.55, 130.14, 137.53, 144.72 (Ph), 172.26 (-CO-).Calc. for $\mathrm{C}_{17} \mathrm{H}_{13} \mathrm{~F}_{6} \mathrm{NO}$ : C, 56.5; H, 3.6; N, 3.8. Found C, 57.00; H, 3.12; N, 4.15.

10) N-(2-methoxybenzyl)-3-(4-methoxyphenyl)-2-propenamide (27)

Yield $(0.11 \mathrm{~g}, 13 \%)$, mp $94^{\circ} \mathrm{C}-96^{\circ} \mathrm{C}$. IR (Diamond, $\left.\mathrm{cm}^{-1}\right): v_{\max } 1552,3075,3261 .{ }^{1} \mathrm{H}$ NMR $\left(300 \mathrm{MHz} ; \mathrm{CDCl}_{3}\right.$; $\left.\mathrm{Me}_{4} \mathrm{Si}\right) \delta 3.62$ (3H, s), $3.71(3 \mathrm{H}, \mathrm{s}), 4.44$ (2H, d, $J=5.9$ ), $6.11(1 \mathrm{H}, \mathrm{brs}, \mathrm{NH}), 6.91$ (2H, ddd, $J=21.2,13.8,4.6$ ), $7.33(8 \mathrm{H}, \mathrm{m}) ;{ }^{13} \mathrm{C}$ NMR $\left(75 \mathrm{MHz} ; \mathrm{CDCl}_{3} ; \mathrm{Me}_{4} \mathrm{Si}\right) \delta 38.14\left(\mathrm{CH}_{2}-\mathrm{Ph}\right), 56.15\left(\mathrm{CH}_{3} \mathrm{O}\right), 111.67(\mathrm{Ph}), 115.69(-\mathrm{CH}=)$, 128.02, 128.80, 129.25, 129.43, 130.38, 139.86 (Ph), 140.24, (-CH=), 158.18, 161.80 (Ph), 166.86 (-CO-). Calc. for $\mathrm{C}_{18} \mathrm{H}_{19} \mathrm{NO}_{3}$ : C, 72.7; H, 6.4; N, 4.7. Found C, 72.82; H, 6.21; N, 5.7.

11) N-(4-fluorobenzyl)-3-(4-nitrophenyl)-2-propenamide (28)

Yield $(0.22 \mathrm{~g}, 14 \%), \mathrm{mp} 180^{\circ} \mathrm{C}-182^{\circ} \mathrm{C}$. IR (Diamond, $\left.\mathrm{cm}^{-1}\right): v_{\max } 1509,3040,3272,1334,1605,1209 .{ }^{1} \mathrm{H}$ NMR (300 MHz; $\left.\mathrm{CDCl}_{3} ; \mathrm{Me}_{4} \mathrm{Si}\right) \delta 4.44(2 \mathrm{H}, \mathrm{d}, J=5.9), 6.91(1 \mathrm{H}, \mathrm{d}, J=15.8), 7.19$ (2H, t, $\left.J=8.9\right), 7.38$ (2H, dd, $J=8.8,5.6), 7.62(1 \mathrm{H}, \mathrm{d}, J=15.9), 7.87(2 \mathrm{H}, \mathrm{d}, J=8.7), 8.30(2 \mathrm{H}, \mathrm{m}), 8.82(1 \mathrm{H}, \mathrm{t}, \mathrm{NH}, J=5.9) ;{ }^{13} \mathrm{C}$ NMR (75 $\mathrm{MHz} ; \mathrm{CDCl}_{3}$; $\left.\mathrm{Me}_{4} \mathrm{Si}\right) \delta 42.48\left(\mathrm{CH}_{2}-\mathrm{Ph}\right), 116.14(\mathrm{Ph}), 125.35(-\mathrm{CH}=), 127.52,129.89,130.66,130.77,136.73$, $138.09(\mathrm{Ph}), 124.86$ (-CH=), $148.92(\mathrm{Ph}), 165.82$ (-CO-).Calc. for $\mathrm{C}_{16} \mathrm{H}_{13} \mathrm{FN}_{2} \mathrm{O}_{3}$ : C, 64.0; H, 4.3; N, 9.3. Found C, 64.36; H, 3.80; N, 9.65.

12) N-(4-fluorobenzyl)-3-(4-phenoxyphenyl)-2-propenamide (29)

Yield $(1.04 \mathrm{~g}, 72 \%), \mathrm{mp} 140^{\circ} \mathrm{C}-142^{\circ} \mathrm{C}$. IR (Diamond, $\left.\mathrm{cm}^{-1}\right): v_{\max } 1458,3044,3274,1151 .{ }^{1} \mathrm{H}$ NMR (300 MHz; $\mathrm{CDCl}_{3}$; $\left.\mathrm{Me}_{4} \mathrm{Si}\right) \delta 4.52$ (2H, d, $J=5.8$ ), $5.97(1 \mathrm{H}, \mathrm{t}, \mathrm{NH}, J=5.0), 6.31(1 \mathrm{H}, \mathrm{d}, J=15.6), 6.99(6 \mathrm{H}, \mathrm{m}), 7.14$ $(1 \mathrm{H}, \mathrm{t}, J=7.4), 7.27(2 \mathrm{H}, \mathrm{m}), 7.35(2 \mathrm{H}, \mathrm{t}, J=7.9), 7.43(2 \mathrm{H}, \mathrm{d}, J=8.7), 7.63(1 \mathrm{H}, \mathrm{d}, J=15.6) ;{ }^{13} \mathrm{C} \mathrm{NMR}(75$ MHz; $\mathrm{CDCl}_{3}$; $\left.\mathrm{Me}_{4} \mathrm{Si}\right) \delta 43.08\left(\mathrm{CH}_{2}-\mathrm{Ph}\right), 115.43,115.64,118.42,118.89(\mathrm{Ph}), 119.56(-\mathrm{CH}=), 123.98,129.39$, 129.88, 134.03 (Ph), 140.86 (-CH=), 156.19, 159.01, 160.96 (Ph), 163.40 (C-F), 165.86 (-CO-). Calc. for $\mathrm{C}_{22} \mathrm{H}_{18} \mathrm{FNO}_{2}$ : C, 76.0; H, 5.2; N, 4.0. Found C, 76.32; H, 4.40, N, 4.34.

13) 3-(3,4-dichlorophenyl)-N-(4-fluorobenzyl)-2-propenamide (30)

Yield $(0.85 \mathrm{~g}, 55 \%), \mathrm{mp} 136^{\circ} \mathrm{C}-138^{\circ} \mathrm{C}$. IR (Diamond, $\left.\mathrm{cm}^{-1}\right): v_{\max } 1553,3075,3247,1131 .{ }^{1} \mathrm{H}$ NMR (300 $\mathrm{MHz}$; $\mathrm{CDCl}_{3}$; $\left.\mathrm{Me}_{4} \mathrm{Si}\right) \delta 6.00$ (1H, brs, $\mathrm{NH}$ ), $6.37(1 \mathrm{H}, \mathrm{d}, J=14.8), 7.01(2 \mathrm{H}, \mathrm{t}, J=8.6), 7.27(3 \mathrm{H}, \mathrm{m}), 7.41(1 \mathrm{H}, \mathrm{d}$, $J=8.3), 7.54(2 \mathrm{H}, \mathrm{t}, J=7.7) ;{ }^{13} \mathrm{C} \mathrm{NMR}\left(75 \mathrm{MHz} ; \mathrm{CDCl}_{3} ; \mathrm{Me}_{4} \mathrm{Si}\right) \delta 43.17\left(\mathrm{CH}_{2}-\mathrm{Ph}\right), 115.50(\mathrm{Ph}), 121.97(-\mathrm{CH}=)$, 126.93, 129.17, 129.51, 130.80, 133.11 (Ph), 133.64, 134.71, (C-Cl), 139.06 (-CH=), 161.00 (Ph), 163.45 (C-F), 164.99 (-CO-); Calc. for $\mathrm{C}_{16} \mathrm{H}_{12} \mathrm{Cl}_{2} \mathrm{FNO}$ : C, 59.2; H, 3.7; 4.3. Found C, 59.62; H, 3.33; N, 4.34.

14) $\mathrm{N}$-[3,5-bis(trifluoromethyl)benzyl]-3-(4-phenoxyphenyl)-2-propenamide (31) 
Yield (0.66 g, 68\%), mp $140^{\circ} \mathrm{C}-142^{\circ} \mathrm{C}$. IR (Diamond, $\mathrm{cm}^{-1}$ ): $v_{\max } 1509,3050,3272,1124 .{ }^{1} \mathrm{H}$ NMR (300 $\mathrm{MHz} ; \mathrm{CDCl}_{3}$; $\left.\mathrm{Me}_{4} \mathrm{Si}\right) \delta 4.68(2 \mathrm{H}, \mathrm{d}, J=6.1), 6.12(1 \mathrm{H}, \mathrm{t}, \mathrm{NH}, J=5.8), 6.35(1 \mathrm{H}, \mathrm{d}, J=15.6), 6.96(2 \mathrm{H}, \mathrm{d}, J=$ 8.6), 7.03 (2H, d, $J=8.6), 7.15$ (1H, t, $J=7.9), 7.25(1 \mathrm{H}, \mathrm{s}), 7.36$ (2H, t $J=7.7), 7.46$ (2H, d, $J=8.7), 7.66(1 \mathrm{H}$, d, $J=15.5), 7.77(2 \mathrm{H}, \mathrm{d}, J=5.4) ;{ }^{13} \mathrm{C} \mathrm{NMR}\left(75 \mathrm{MHz} ; \mathrm{CDCl}_{3}\right.$; $\left.\mathrm{Me}_{4} \mathrm{Si}\right) \delta 42.83\left(\mathrm{CH}_{2}-\mathrm{Ph}\right), 118.05,118.41(\mathrm{Ph})$, 119.61 (-CH=), 121.46 (C-F), 124.06, 127.77, 129.16, 129.56, 129.53, 129.90, 131.78, 132.12 (Ph), 141.08 (-CH=), 141.74, 156.11, 159.26 (Ph), 166.16 (-CO-).Calc. for $\mathrm{C}_{24} \mathrm{H}_{17} \mathrm{~F}_{6} \mathrm{NO}_{2}$ : C, 61.9; H, 3.6; N, 3.0 Found C, 62.12; H, 2.64; N, 3.43 .

15) $\mathrm{N}$-(4-fluorobenzyl)-3-(4-methylphenyl)-2-propenamide (32)

Yield $(0.49 \mathrm{~g}, 49 \%), \mathrm{mp} 144^{\circ} \mathrm{C}-146^{\circ} \mathrm{C}$. IR (Diamond, $\mathrm{cm}^{-1}$ ): $v_{\max } 1509,3036,3261,1209 .{ }^{1} \mathrm{H}$ NMR (300 $\left.\mathrm{MHz} ; \mathrm{CDCl}_{3} ; \mathrm{Me}_{4} \mathrm{Si}\right) \delta 2.34(3 \mathrm{H}, \mathrm{s}), 4.51(2 \mathrm{H}, \mathrm{d}, J=5.8), 6.03(1 \mathrm{H}, \mathrm{brs}, \mathrm{NH}), 6.36(1 \mathrm{H}, \mathrm{d}, J=15.6), 6.99(2 \mathrm{H}, \mathrm{t}$, $J=8.7), 7.14(2 \mathrm{H}, \mathrm{d}, J=7.9), 7.27(2 \mathrm{H}, \mathrm{m}), 7.37(2 \mathrm{H}, \mathrm{d}, J=8.1), 7.62(1 \mathrm{H}, \mathrm{d}, J=15.6) ;{ }^{13} \mathrm{C} \mathrm{NMR}(75$ $\left.\mathrm{MHz} ; \mathrm{CDCl}_{3} ; \mathrm{Me}_{4} \mathrm{Si}\right) \delta 21.38\left(\mathrm{CH}_{3}\right), 43.04\left(\mathrm{CH}_{2}-\mathrm{Arom}\right), 115.40(\mathrm{Ph}), 119.15(-\mathrm{CH}=), 127.75,129.52,131.90$, 134.08, 140.07 (Ph), 141.51 (-CH=), 160.93 (C-F), 163.38 (Ph), 165.97 (-CO-).Calc. for $\mathrm{C}_{17} \mathrm{H}_{16} \mathrm{FNO}$ : C, 75.8; H, 5.9; N, 5.2. Found C, 76.29; H, 5.23; N, 5.39.

\section{Acknowledgements}

The authors would like to acknowledge PAPIIT/UNAM Projects No. IN200614 and IT202015 and project PIAPI VC02 for partially supporting this work and F. Sotres, D. Jiménez, M. Duarte and Rosa Ma. Valadez from FESC-UNAM for their skillful technical assistance. This work was conducted as a part of the Project Cátedra: Diseño de Sustancias Bioactivas of FESC-UNAM-2012.

\section{References}

[1] Demestre, M., Messerli, S.M., Celli, N., Shahhossini, M., Kluwe, L., Mautner, V. and Maruta, H. (2008) CAPE (Caffeic Acid Phenethyl Ester)-Based Propolis Extract (Bio 30) Suppresses the Growth of Human Neurofibromatosis (NF) Tumor Xenografts in Mice. Phytotherapy Research, 23, 226-230. http://dx.doi.org/10.1002/ptr.2594

[2] Natarajan, K., Singh, S., Burke, T.R., Grunberger, D. and Aggarwal, B.B. (1996) Caffeic Acid Phenethyl Ester Is a Potent and Specific Inhibitor of Activation of Nuclear Transcription Factor NF-Kappa B. Proceedings of the National Academy of Sciences of the United States of America, 93, 9090-9095. http://dx.doi.org/10.1073/pnas.93.17.9090

[3] Orban, Z., Mitsiades, N., Burke, T.R., Tsokos, M. and Chrousos, G.P. (2000) Caffeic Acid Phenethyl Ester Induces Leukocyte Apoptosis, Modulates Nuclear Factor-Kappa B and Suppresses Acute Inflammation. Neuroimmunomodulation, 7, 99-105. http://dx.doi.org/10.1159/000026427

[4] Huang, M.T., Ma, W., Yen, P., Xie, J.G., Han, J., Frenkel, K., Grunberger, D. and Conney, A.H. (1996) Inhibitory Effects of Caffeic Acid Phenethyl Ester (CAPE) on 12-O-Tetradecanoylphorbol-13-Acetate-Induced Tumor Promotion in Mouse Skin and The Synthesis of DNA, RNA and Protein in Hela Cells. Carcinogenesis, 17, 761-765. http://dx.doi.org/10.1093/carcin/17.4.761

[5] Huang, M.T., Smart, R.C., Wong, C.Q. and Conney, A.H. (1988) Inhibitory Effect of Curcumin, Chlorogenic Acid, Caffeic Acid, and Ferulic Acid on Tumor Promotion in Mouse Skin by 12-O-Tetradecanoylphorbol-13-Acetate. Cancer Research, 48, 5941-5946.

[6] Rajan, P., Vedernikova, I., Cos, P., Berghe, D.V., Augustyns, K. and Haemers, A. (2001) Synthesis and Evaluation of Caffeic Acid Amides as Antioxidants. Bioorganic \& Medicinal Chemistry Letters, 11, 215-217. http://dx.doi.org/10.1016/S0960-894X(00) 00630-2

[7] Naito, Y., Sugiura, M., Yamaura, Y., Fukaya, C., Yokoyama, K., Nakagawa, Y., Ikeda, T., Senda, M. and Fujita, T. (1991) Quantitative Structure-Activity Relationship of Catechol Derivatives Inhibiting 5-Lipoxygenase. Chemical and Pharmaceutical Bulletin, 39, 1736-1745. http://dx.doi.org/10.1248/cpb.39.1736

[8] Son, S. and Lewis, B.J. (2002) Free Radical Scavenging and Antioxidative Activity of Caffeic Acid Amide and Ester Analogues: Structure-Activity Relationship. Journal of Agricultural and Food Chemistry, 50, 468-472. http://dx.doi.org/10.1021/jf010830b

[9] Nishioka, T., Watanabe, J., Kawabata, J. and Niki, R. (1997) Isolation and Activity of N-p-Coumaroyltyramine, an $\alpha$ Glucosidase Inhibitor in Welsh Onion (Allium fistulosum). Bioscience, Biotechnology, and Biochemistry, 61, 11381141. http://dx.doi.org/10.1271/bbb.61.1138

[10] Macias-Perez, J.R., Beltrán-Ramírez, O., Vásquez-Garzón, V.R., Salcido-Neyoy, M.E., Martínez-Soriano, P.A., Ruiz-Sanchez, M.B., Ángeles E. and Villa-Treviño, S. (2013) The Effect of Caffeic Acid Phenethyl Ester Analogues in a Modified Resistant Hepatocyte Model. Anti-Cancer Drugs, 24, 394-405.

http://dx.doi.org/10.1097/CAD.0b013e32835e9743 
[11] Plagens, A. and Laue, T. (2005) Named Organic Reactions. 2nd Edition, John Wiley \& Sons, New York, 320.

[12] Wolff, H. (1946) The Schmidt Reaction. Organic Reactions, 3, 307-336.

[13] Chandrasekhar, S. and Gopalaiah, K. (2003) Ketones to Amides via a Formal Beckmann Rearrangement in “One Pot”: A Solvent-Free Reaction Promoted by Anhydrous Oxalic Acid. Possible Analogy with the Schmidt Reaction. Tetrahedron Letters, 44, 7437-7439. http://dx.doi.org/10.1016/j.tetlet.2003.08.038

[14] Yadav, J.S., Reddy, B.V.S., Reddy, U.V.S. and Praneeth, K. (2008) Azido-Schmidt Reaction for the Formation of Amides, Imides and Lactams from Ketones in the Presence of $\mathrm{FeCl}_{3}$. Tetrahedron Letters, 49, 4742-4745. http://dx.doi.org/10.1016/j.tetlet.2008.05.113

[15] Lee, H.-L. and Aubé, J. (2007) Intramolecular and Intermolecular Schmidt Reactions of Alkyl Azides with Aldehydes. Tetrahedron, 63, 9007-9015. http://dx.doi.org/10.1016/j.tet.2007.05.079

[16] Liang, J., Jing, L. and Shang, Z.C. (2011) Metal-Free Synthesis of Amides by Oxidative Amidation of Aldehydes with Amines in PEG/Oxidant System. Tetrahedron, 67, 8532-8535. http://dx.doi.org/10.1016/j.tet.2011.08.091

[17] Zhang, M. and Wu, X.F. (2013) Zinc(II)-Catalyzed Oxidative Amidation of Arylaldehydes with Alkylamines under Solvent-Free Conditions. Tetrahedron Letters, 54, 1059-1062. http://dx.doi.org/10.1016/j.tetlet.2012.12.010

[18] Suto, Y., Yamagiwa, N. and Torisawa, Y. (2008) Pd-Catalyzed Oxidative Amidation of Aldehydes with Hydrogen Peroxide. Tetrahedron Letters, 49, 5732-5735. http://dx.doi.org/10.1016/j.tetlet.2008.07.075

[19] Organic Chemistry Portal 2008. www.organic-chemistry.org

[20] Strukil, V., Bartolec, B., Portada, T., Dilovic, I., Halasz, I. and Margetic, D. (2012) One-Pot Mechanosynthesis of Aromatic Amides and Dipeptides from Carboxylic Acids and Amines. Chemical Communications, 48, 12100-12102.

[21] Gooßen, L.J., Ohlmann, D.M. and Lange, P.P. (2009) The Thermal Amidation of Carboxylic Acids Revisited. Synthesis, 2009, 160-164. http://dx.doi.org/10.1055/s-0028-1083277

[22] Hosseini, M., Sodagar, E. and Doroodmand, M.M. (2011) Nano Sulfated Titania as Solid Acid Catalyst in Direct Synthesis of Fatty Acid Amides. The Journal of Organic Chemistry, 76, 2853-2859. http://dx.doi.org/10.1021/jo2002769

[23] Dunetz, J., Xiang, Y., Baldwin, A. and Ringling, J. (2011) General and Scalable Amide Bond Formation with Epimerization-Prone Substrates Using T3P and Pyridine. Organic Letters, 13, 5048-5051.

[24] Huang, Z.P., Reilly, J.R. and Buckle, R.N. (2007) An Efficient Synthesis of Amides and Esters via Triacyloxyboranes. Synlett, 2007, 1026-1030. http://dx.doi.org/10.1055/s-2007-973890

[25] Chung, S., Uccello, D.P., Choi, H., Montgomery, J.I. and Chen, J. (2011) Trimethylaluminium-Facilitated Direct Amidation of Carboxylic Acids. Synlett, 2011, 2072-2074.

[26] Khalafi-Nezhad, A., Parhami, A., Rad, M.N.S. and Abdolkarim, Z. (2005) Efficient Method for the Direct Preparation of Amides from Carboxylic Acids Using Tosyl Chloride under Solvent-Free Conditions. Tetrahedron Letters, 46, 6879-6882. http://dx.doi.org/10.1016/j.tetlet.2005.08.021

[27] Khalafi-Nezhad, A., Mokhtari, B. and Rad, M.N.S. (2003) Direct Preparation of Primary Amides from Carboxylic Acids and Urea Using Imidazole under Microwave Irradiation. Tetrahedron Letters, 44, 7325-7328.

[28] Lannuzel, M., Lamothe, M. and Perez, M. (2001) An Efficient One-Pot, Purification-Free, Preparation of Amides Using Polymer-Supported Reagents. Tetrahedron Letters, 42, 6703-6705. http://dx.doi.org/10.1016/S0040-4039(01)01387-9

[29] Shaabani, A., Soleimani, E. and Rezayan, A.H. (2007) A Novel Approach for the Synthesis of Aryl Amides. Tetrahedron Letters, 48, 6137-6141. http://dx.doi.org/10.1016/j.tetlet.2007.06.136

[30] Huang, P., Zheng, X. and Deng, X. (2001) DIBAL-H-H ${ }_{2}$ NR and DIBAL-H-HNR ${ }^{1} \mathrm{R}^{2} \cdot \mathrm{HCl}_{\text {Complexes for Efficient }}$ Conversion of Lactones and Esters to Amides. Tetrahedron Letters, 42, 9039-9041. http://dx.doi.org/10.1016/S0040-4039(01)01933-5

[31] Ghaffarzadeh, M., Joghan, S.S. and Faraji, F. (2012) A New Method for the Synthesis of Amides from Imines. Tetrahedron Letters, 53, 203-206. http://dx.doi.org/10.1016/j.tetlet.2011.11.018

[32] Kim, B., Lee, H.G., Kang, S.B., Sung, G.H., Kim, J.J., Park, J.K., Lee, S.G. and Yoon, Y.J. (2012) Tert-Butoxide-Assisted Amidation of Esters under Green Conditions. Synthesis, 2012, 42-50.

[33] Veitch, G., Bridgwood, K.L. and Ley, S.V. (2008) Magnesium Nitride as a Convenient Source of Ammonia: Preparation of Primary Amides. Organic Letters, 10, 3623-3625.

[34] Cao, L., Ding, J., Gao, M., Wang, Z., Li, J. and Wu, A. (2009) Novel and Direct Transformation of Methyl Ketones or Carbinols to Primary Amides by Employing Aqueous Ammonia. Organic Letters, 11, 3810-3813.

[35] Sinha, A.K., Sharma, A. and Joshi, B.P. (2007) One-Pot Two-Step Synthesis of 4-Vinylphenols from 4-Hydroxy Substituted Benzaldehydes under Microwave Irradiation: A New Perspective on the Classical Knoevenagel-Doebner Reaction. Tetrahedron, 63, 960-965. http://dx.doi.org/10.1016/j.tet.2006.11.023 
[36] Wu, X.-A., Ying, P., Liu, J.-Y., Shen, H.-S., Chen, Y. and He, L. (2009) Lithium Chloride-Assisted Selective Hydrolysis of Methyl Esters under Microwave Irradiation. Synthetic Communications, 39, 3459-3470.

[37] Phillips, A.P. (1954) The Condensation of Aromatic Aldehydes with 2-Ethylpyridine and with 4-Ethylpyridine. Journal of the American Chemical Society, 76, 3986-3987. http://dx.doi.org/10.1021/ja01644a036

[38] Szymanski, W., Wu, B., Weiner, B., de Wildeman, S., Feringa, B.L. and Janssen, D.B. (2009) Phenylalanine Aminomutase-Catalyzed Addition of Ammonia to Substituted Cinnamic Acids: A Route to Enantiopure $\alpha$ - and $\beta$-Amino Acids. The Journal of Organic Chemistry, 74, 9152-9157.

[39] Singh, G., Bali, S. and Singh, A.K. (2007) Palladium(0) Complexes of (P,P) and (P,N) Ligands Immobilized on Silica Gel as Catalysts in Selective Heck Type Carbon-Carbon Coupling Reactions. Polyhedron, 26, 897-903. http://dx.doi.org/10.1016/i.poly.2006.09.043

[40] Brittelli, D.R. (1981) Phosphite-Mediated in Situ Carboxyvinylation: A New General Acrylic Acid Synthesis. The Journal of Organic Chemistry, 46, 2514-2520.

[41] Fukuyama, T., Arai, M., Matsubara, H. and Ryu, I. (2004) Mizoroki-Heck Arylation of $\alpha$, $\beta$-Unsaturated Acids with a Hybrid Fluorous Ether, F-626: Facile Filtrative Separation of Products and Efficient Recycling of a Reaction Medium Containing a Catalyst. The Journal of Organic Chemistry, 69, 8105-8107. http://dx.doi.org/10.1021/jo049028+

[42] Zhu, M., Song, Y.L. and Cao, Y. (2007) A Fast and Convenient Heck Reaction in Water under Microwave Irradiation. Synthesis, 2007, 853-856. http://dx.doi.org/10.1055/s-2007-965936

[43] Watanabe, T., Hayashi, K., Yoshimatsu, S. and Sakai, K. (1980) Studies of Hypolipidemic Agents. 1. Synthesis and Hypolipidemic Activities of Alkoxycinnamic acid Derivatives. Journal of Medicinal Chemistry, 23, 50-59.

[44] Kawamatsu, Y., Asakawa, H., Saraie, T., Imamiya, E., Nishikawa, K. and Hamuro, Y. (1980) Studies on Antihyperlipidemic Agents. II. Synthesis and Biological Activities of 2-Chloro-3-arylpropionic Acids. Arzneimittel Forschung, 30, 585-589.

[45] Ganushchak, N.I., Fedorovich, I.S., Obushak, N.D. and Prokopishin, I.Y. (1982) Reaction of Methyl Esters of Acrylic and Methacrylic Acids with Bisdiazotizedbenzidines and Their Analogs. Zhurnal Organicheskoi Khimii, 18, 17271732.

[46] King, F.D. and Caddick, S. (2013) The Triflic Acid-Mediated Cyclisation of $N$-Benzyl-Cinnamamides. Tetrahedron, 69, 487-491. http://dx.doi.org/10.1016/j.tet.2012.11.035

[47] Lundberg, H., Fredrik, T. and Hans, A. (2012) Titanium(IV) Isopropoxide as an Efficient Catalyst for Direct Amidation of Nonactivated Carboxylic Acids. Synlett, 23, 2201-2204.

[48] Iranpoor, N., Firouzabadi, H., Motevalli, S. and Talebi, M. (2013) Palladium-Free Aminocarbonylation of Aryl, Benzyl, and Styryl Iodides and Bromides by Amines Using $\mathrm{Mo}(\mathrm{CO})_{6}$ and Norbornadiene. Tetrahedron, 69, 418-426.

[49] Wu, Y.J., He, H., Sun, L.Q., L’Heureux, A., Chen, J., Dextraze, P., Starrett Jr., J.E., Boissard, C.G., Gribkoff, V.K., Natale, J. and Dworetzky, S.I. (2004) Synthesis and Structure-Activity Relationship of Acrylamides as KCNQ2 Potassium Channel Openers. Journal of Medicinal Chemistry, 47, 2887-2896.

[50] Chen, C.-C., Ho, J.-C. and Chang, N.-C. (2008) A Practical and Efficient Synthesis of (E)- $\beta$-aryl- $\alpha, \beta$-Unsaturated Amides. Tetrahedron, 64, 10350-10354.

[51] Pardin, C., Pelletier, J.N., Lubell, W.D. and Keillor, J.W. (2008) Cinnamoyl Inhibitors of Tissue Transglutaminase. The Journal of Organic Chemistry, 73, 5766-5775. http://dx.doi.org/10.1021/jo8004843

[52] Fu, Z.Q., Lee, J., Kang, B. and Hong, S.H. (2012) Dehydrogenative Amide Synthesis: Azide as a Nitrogen Source. Organic Letters, 14, 6028-6031. http://dx.doi.org/10.1021/ol302915g

[53] Metro, T.X., Bonnamour, J., Reidon, T., Sarpoulet, J., Martinez, J. and Lamaty, F. (2012) Mechanosynthesis of Amides in the Total Absence of Organic Solvent from Reaction to Product Recovery. Chemical Communications, 48, 11781-11783. http://dx.doi.org/10.1039/c2cc36352f

[54] Thalluri, K., Nadimpally, K.C., Chakravarty, M.P., Paul, A. and Mandal, B. (2013) Ethyl 2-(tert-Butoxycarbonyloxyimino)2-cyanoacetate (Boc-Oxyma) as Coupling Reagent for Racemization-Free Esterification, Thioesterification, Amidation and Peptide Synthesis. Advanced Synthesis \& Catalysis, 355, 448-462. http://dx.doi.org/10.1002/adsc.201200645

[55] Barajas, J.G.H., Mendez, L.Y.V., Kouznetsov, V.V. and Stashenko, E.E. (2008) Efficient Synthesis of New N-Benzylor $N$-(2-Furylmethyl)cinnamamides Promoted by the "Green" Catalyst Boric Acid, and Their Spectral Analysis. Synthesis, 2008, 377-382.

[56] Pizzetti, M., Russo, A. and Petricci, E. (2011) Microwave-Assisted Aminocarbonylation of Ynamides by Using Catalytic $\left[\mathrm{Fe}_{3}(\mathrm{CO})_{12}\right]$ at Low Pressures of Carbon Monoxide. Chemistry-A European Journal, 17, 4523-4528. http://dx.doi.org/10.1002/chem.201100447

[57] Wu, J., Liu, Y. and Dai, W. (2008) Faming Zhuanli Shenqing Gongkai Shuomingshu. CN 101274901. 